\title{
Cycle évolutif d'un Dicheilonematinae, Serratospiculum tendo, Filaire parasite du faucon
}

\author{
par O. BAIN et G. VASSILIADES
}

[Laboratoire de Zoologie (Vers), associé au C.N.R.S., Muséum National d'Histoire Naturelle]

\section{Résumé}

Le cycle larvaire de la Filaire Serratospiculum tendo (Dicheilonematinea) a été réalisé chez Locusta migratoria; il est analogue à ceux des Diplotriaeninae et confirme à nouveau les ressemblances biologiques qui existent entre les Diplotriaenidae et les Spirurides primitifs. Chez la larve infestante de $S$. tendo, les caractères morphologiques ancestraux (bouche hexagonale, papilles labiales internes présentes) sont bien conservés.

\section{Summary}

The larval cycle of the Filarioid worm Serratospiculum tendo (Dicheilonematinea) was reproduced in Locusta migratoria; it is comparable to those of Diplotriaeninae and emphasizes again the existence of biological similarities between the Diplotriaenidae and the primitive Spirurides. In the infective larva of $S$. tendo, the ancestral morphological characteristics (hexagonal mouth, presence of labial papillae) are well preserved.

\section{Introduction.}

La biologie des Filaires ovipares est restée longtemps énigmatique ; la présence de ces Nématodes dans des organes réellement ou apparement sans communication avec le milieu extérieur posait un important problème de transmission, qui a été résolu il y a une dizaine d'années.

Pour les Filaires ovipares d'Oiseaux et de Reptiles, localisées ailleurs que dans le tissu sous-cutané ou la cavité orbitaire, le problème a été graduellement élucidé : Blanc, 
(1919) remarque que les Diplotriaena sont situés dans les sacs aériens des Oiseaux et non dans la cavité générale; Chabaud (1955), expérimentant sur deux espèces de Diplotriaena, démontre que les œufs sont expulsés par voie proctodéale, après avoir été drainés par les bronches, et prévoit que le cycle larvaire doit ressembler à celui d'un Spiruridae ; Anderson (1957) réalise le premier cycle de Filaire ovipare d'Oiseau, avec Diplotriaenoïdes translucidus Anderson, 1956; l'hôte intermédiaire est un Orthoptère. Plus récemment (1962) le même auteur effectue un nouveau cycle de Diplotriaeninae, il confirme, à nouveau, les ressemblances biologiques de ce groupe avec les Spirurides. Mais si deux cyc'es de Diplotriaeninae ont été élucidés, aucun cycle n'a encore été effectué chez les Dicheilonematinae, les Desmidocercidae et les Aproctidae.

Ayant eu la chance de recevoir des spécimens vivants de Serratospiculum tendo (Nitzsch, 1857) (Dicheilonematinae) * il a donc paru particulièrement intéressant d'en tenter le cycle.

Il s'avère très proche des cycles de Diplotriaeninae.

\section{Origine du matériel et infestations expérimentales.}

Un fragment de tissu pu'monaire de Falco peregrinus, recueilli par le $\mathrm{D}^{r}$ Vallée, nous a été très aimablement envoyé par le $\mathrm{P}^{\mathrm{r}}$ Lamy (Institut Pasteur); il contenait quatre spécimens vivants de Serratospiculum tendo * (2 $\delta^{\top}$ et 2 \%).

Les $\delta$ et les extrémités des $q$ ont été fixées ; les utérus bour:és d'œufs embryonnés, ont permis d'infester divers Insectes, fournis par le Vivarium du M. N. d'H. N. grâce à l'obligeance de M. Planchard : Locusta migratoria (L., 1778) (trois spécimens), Geotrupes sylvaticus Panz (cinq spécimens), Periplaneta americana (L., 1758) (dix spécimens), Tenebrio molitor (L., 1758) (cinq larves); seules les deux premières espèces ont été infestées individuel'ement.

Parmi ces quatre espèces, deux seulement se sont révélées positives: les Locustes et les Geotrupes; il est possible que les Blattes et les Tenebrio, apparemment négatifs, n'aient pas ingéré d'œufs.

Un coq a reçu par voie orale vingt-cinq larves infestantes et les restes d'une Locuste positive. Il a été disséqué quinze jours plus tard ; aucune larve ni aucun granulome n'ont été mis en évidence.

* Nous identifions nos spécimens à Serratospiculum tendo parce que leur morphologie, et principalement la tête et la queue du $\delta^{\alpha}$, sont conformes aux figures données par Yorke et Maplestone en 1926 et par Tubangui en 1934 (l'espèce décrite par cet auteur sous le nom de $S$. thoracis, a été mise en synonymie avec $S$. tendo par Sonin en 1968). Etant donnée l'incertitude qui règne encore dans la systématique du genre nous préférons donner la description des spicules (fig. 1) qui sont complexes et doivent avoir une grande valeur spécifique. Le spicule gauche long de $1150 \mu$, comprend un manche fortement sclérifié, long de $265 \mu$, auquel fait suite une lame très développée et complexe; elle comprend d'abord une portion proximale, longue de $600 \mu$, constituée par un axe dorsal sclérifié et ponctué prolongé par deux ailes membraneuses repliées l'une vers l'autre; l'aile gauche est la plus large et est ornée sur sa face interne par deux groupes longitudinaux d'épines; l'aile droite ne porte qu'un groupe d'épines; la pointe de chaque épine est dirigée vers le manche; la partie distale de la lame est étroite et forme une gouttière membraneuse plissée dont les arêtes sont bien sclérifiées (fig. 1 A et B). Le spicule droit, long de $510 \mu$, comprend un manche épais ( $200 u$ de long) et une lame très sclérifiée ; l'ensemb'e subit une torsion en formə d'S ; la lame est creusée en gouttière et est renforcée sur sa face externe par 10 côtes transversales, particulièrement saillantes vers la région distale (fig. 1 C et D). 


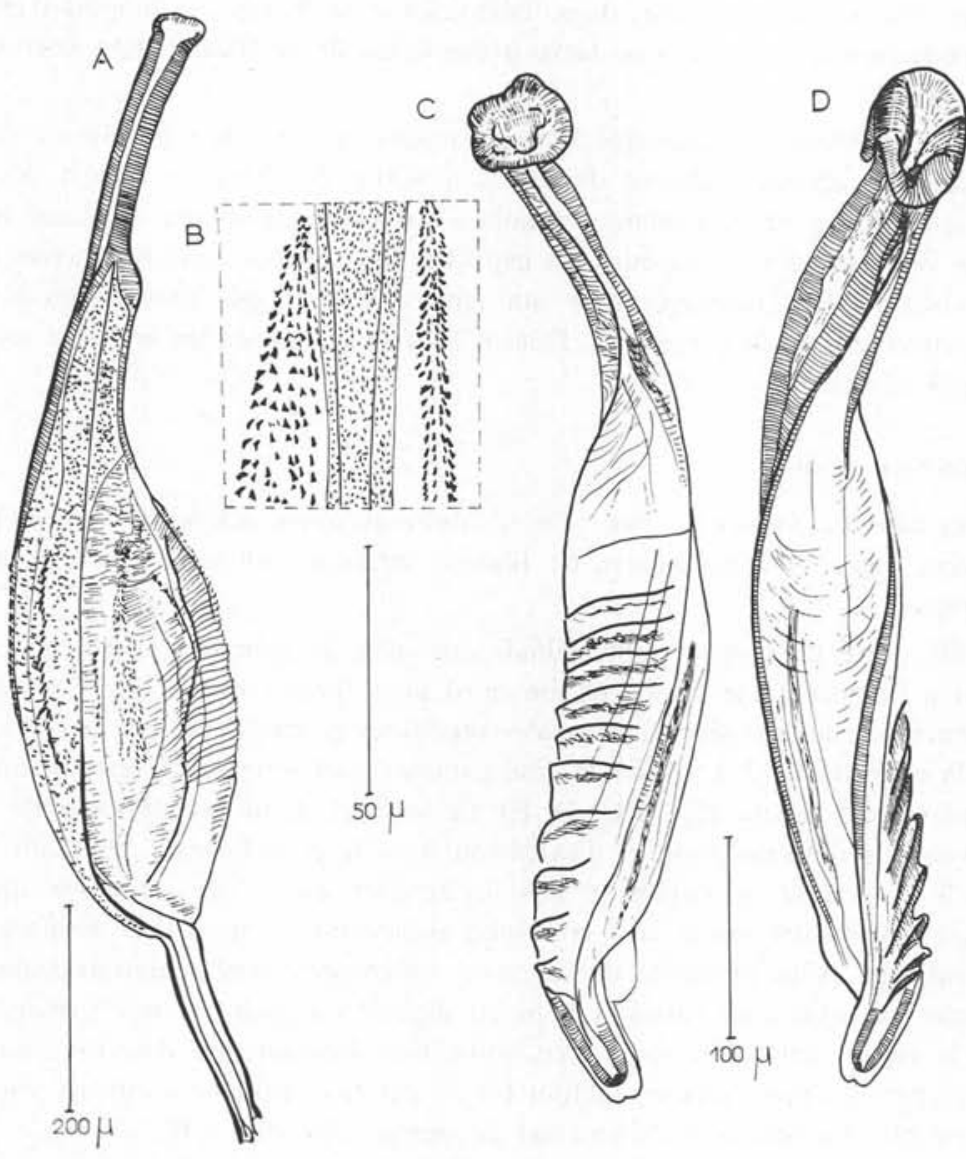

FIG. 1. - Spicules de l'adulte $\delta$ : A) spicule gauche, vue interne droite; B) idem, ornementations cuticulaires internes; C) spicule droit, vue latérale droite ; D) idem, vue interne latérale gauche (A, éch. $200 \mu$; B, éch. $50 \mu$; C et $\mathrm{D}$, éch. $100 \mu$ )

Allure du DÉVeloppement.

Le développement s'est effectué en vingt-quatre jours, à une température variant entre $25^{\circ}$ et $28^{\circ} \mathrm{C}$; il a été suivi chez les Locustes.

Un criquet disséqué le sixième jour permet de recueillir une larve légèrement mobile, en mue I, ayant les caractères cuticulaires céphaliques et caudaux de la larve à l'éclosion, mais présentant un début d'exuviation aux extrémités du corps ; durant le premier stade la larve s'est à peine allongée mais s'est épaissie.

Un deuxième criquet disséqué six jours plus tard libère très rapidement un grand nombre de larves, ce qui permet de supposer qu'elles ne sont pas encapsulées; ces 
larves sont peu mobiles et situées dans l'abdomen et le thorax; la plupart d'entre elles sont au deuxième stade, mais une larve présente un début d'exuviation correspondant à la mue II.

Le dernier criquet est disséqué le vingt-quatrième jour ; il renferme de très nombreuses capsules épaisses, claires, de $600 \mu$ à $800 \mu$ de diamètre (fig. $4 \mathrm{~A}$ ), chaque capsule contient une larve infestante immobile, lovée sur elle-même, la queue au centre et la face ventrale vers l'extérieur; les capsules sont situées dans les travées du tissu adipeux abdominal et thoracique, ce qui laisse supposer que tout le développement larvaire s'effectue dans le corps gras. Durant le troisième stade les larves se sont considérablement al ongées.

\section{ETUdE MORPHOLOGIQUe.}

LARVE NOUVELLEMENT ÉCLOSE : De nombreuses larves écloses et bien mobiles ont été récoltées, dans l'intestin moyen de Blattes, infestées individuellement trois heures après le repas.

La tête porte un crochet subterminal, situé dans le plan latéral gauche; de face, le crochet a l'aspect d'une écaille et son bord libre forme trois pointes; en arrière la tête est ornée par des rangées transversales régulières de très petits points (17 à 19 rangées sur le côté droit, 13 à 15 sur le côté gauche); ces rangées s'interrompent dans le plan latéro-ventral gauche (fig. $2 \mathrm{C}, \mathrm{D}, \mathrm{E}$ ). Le sommet de la tête, très mobile, effectue des mouvements d'invagination et d'extension dans le plan latéral: les tissus s'invaginent sur le côté droit et ramènent ainsi le crochet dans l'axe du corps (fig. 2 B). L'extrémité postérieure porte une couronne subterminale de petites pointes, et une pointe terminale. Ailes présentes débutant en arrière de l'ornementation céphalique et se terminant au niveau de l'anus. L'appareil digestif est déjà organisé : œsophage peu net dans la région antérieure mais avec bulbe bien individualisé, intestin creux contenant les déchets du métabolisme, rectum formé par trois cellules à noyaux volumineux disposés en file. La cellule excrétrice est de petite taille (fig. 2 B).

Dimensions : $250 \mu$ de long, $14 \mu$ de large; anneau nerveux et pore excréteur respectivement à $50 \mu$ et $92 \mu$ de l'apex; œsophage long de $135 \mu$; queue longue de $32 \mu$.

Premier stade larvaire : Il est connu seulement par une larve en mue I (fig. 2 G). Le corps est court et épais, recouvert d'une cuticule très fine ornée de stries transversales qui s'interrompent au niveau des cordes latérales (fig. 2 F). La couche épitheliomusculaire est épaisse. Les structures cellulaires ne sont pas nettes sur le vivant. Dans la région antérieure, l'exuvie de la mue I entraîne avec elle un axe cuticulaire cesophagien; la région buccale du très jeune stade II est invaginée ; entre elle et l'œsophage s'intercale un segment très faib'ement cuticularisé. L'œsophage a une structure qui est proche du type rhabditoïde: bulbe large mais dépourvu de valvule, isthme étroit, corpus rétréci au niveau de l'anneau nerveux. La cellu'e excrétrice est très volumineuse et les deux canaux excréteurs sont bien visibles sous la cuticule du stade I ; la queue présente une pointe arrondie sans ornementations cuticulaires; le bouchon cuticulaire 


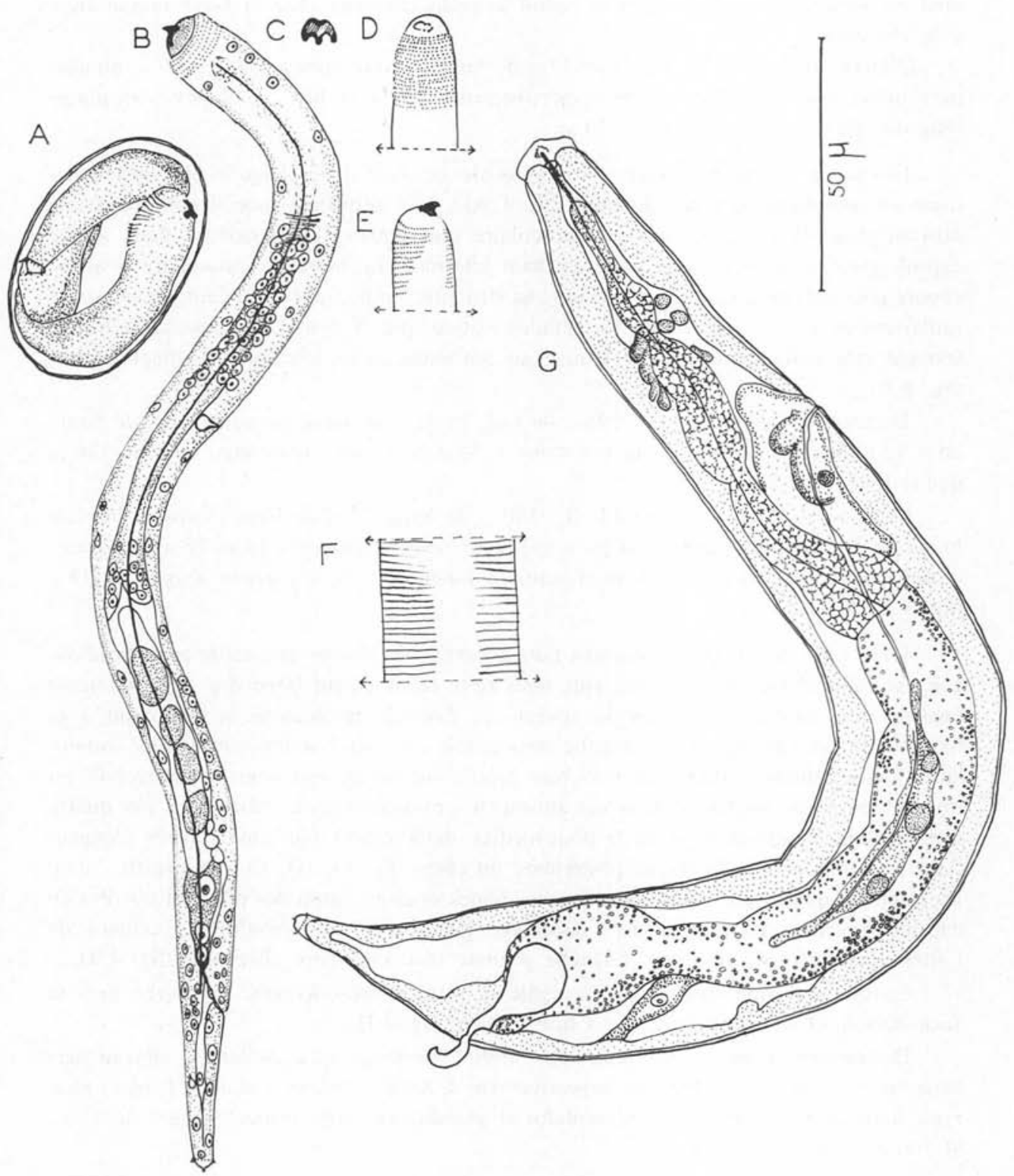

FIG. 2. - A) larve dans l'œuf ; B) larve nouvellement éclose ; C) idem, crochet subterminal, vue de face; D) idem, région céphalique, vue latérale droite; E) idem, vue latérale gauche; F) larve en mue I, ornementation cuticulaire, vue latérale; G) larve à la fin du $1^{\text {er }}$ stade. (Ech. $50 \mu$, sauf C, à main-levée) 
anal est saillant et vésiculeux (il se réduit considérablement chez la larve immobilisée à la chaleur).

Dimensions : $270 \mu$ de long sur $33 \mu$ de large ; pré-œsophage haut de $9 \mu$, anneau nerveux et pore excréteur situés respectivement à $39 \mu$ et $68 \mu$ de l'apex ; œsophage long de $102 \mu$; queue longue de $39 \mu$.

DeuXiÈme Stade LARVAire: Au cours de ce stade l'œsophage évolue graduellement en œsophage de type spiruride (fig. $3 \mathrm{~A}$ ); à la deuxième mue il comprend une portion glandulaire et une portion musculaire plus étroite. Le deuxième stade a une capsule buccale dont la base seule est bien sclérifiée. Le bouchon cuticulaire anal est encore plus saillant qu'au premier stade ; sa structure est homogène au centre mais striée radiairement à son contact avec les cellules rectales (fig. $3 \mathrm{~A}$ et $\mathrm{D}$ ). L'ébauche génitale, souvent très malaisée à voir, se trouve au voisinage de la jonction œsophage-intestin (fig. 3 B).

Dimensions d'un stade II : $295 \mu$ de long et $32 \mu$ de large ; capsule buccale haute de $8 \mu$; anneau nerveux et pore excréteur à $50 \mu$ de l'apex; œsophage long de $116 \mu$, queue longue de $20 \mu$.

Dimensions d'une larve en M. II : $380 \mu$ de long, $37 \mu$ de large ; capsule buccale haute de $5 \mu$; anneau nerveux et pore excréteur respectivement à 50 et $55 \mu$ de l'apex ; œsophage long de $162 \mu$ (portion musculaire longue de $70 \mu$ ); queue longue de $18 \mu$ (fig. $3 \mathrm{E}, \mathrm{F}, \mathrm{G})$.

DÉbut DU STADE III ET FORMES INFESTANTES : La forme infestante est très allongée par rapport aux stades précédents mais reste relativement large (fig. 4 B). Cuticule épaisse, ailes latérales débutant au niveau de l'anneau nerveux et se terminant à la hauteur du rectum (fig. $4 \mathrm{G}$ ). Bouche hexagonale au centre d'une mince lame cuticulaire; deux pointes médianes dont la base repose sur un anneau sclérifié hexagonal ; au niveau des quatre angles latéraux cet anneau se prolonge dans le même plan par quatre axes également sclérifiés ; dans le plan médian deux autres axes cuticularisés plongent dans le tégument vers la région postérieure du corps (fig. $4 \mathrm{C}, \mathrm{D}, \mathrm{E}$ ); six papilles labiales internes, quatre grosses papilles sub-médianes et deux amphides plus petites. Pas de capsule buccale; court pharynx; œsophage glandulaire très développé; cellules de l'intestin chargées de réserves; ébauche génitale très antérieure chez la q (fig. 4 B).

La queue a une base large, puis elle se rétrécit brusquement, se courbe vers la face dorsale et se termine par six à huit pointes (fig. $4 \mathrm{H}$ ).

Dimensions d'une forme infestante : $1090 \mu$ de long ; $62 \mu$ de large ; anneau nerveux, pore excréteur et deirides respectivement à $84 \mu, 104 \mu$ et $116 \mu$ de l'apex ; pharynx haut de $10 \mu$; œsophage musculaire et glandulaire longs respectivement de $150 \mu$ et $500 \mu$; queue longue de $32 \mu$.

\section{Conclusion.}

1) Origine des ornementations céphaliques de l'adulte chez Serratospiculum tendo. Il est très probable que le cadre cuticulaire céphalique de la larve du troisième 


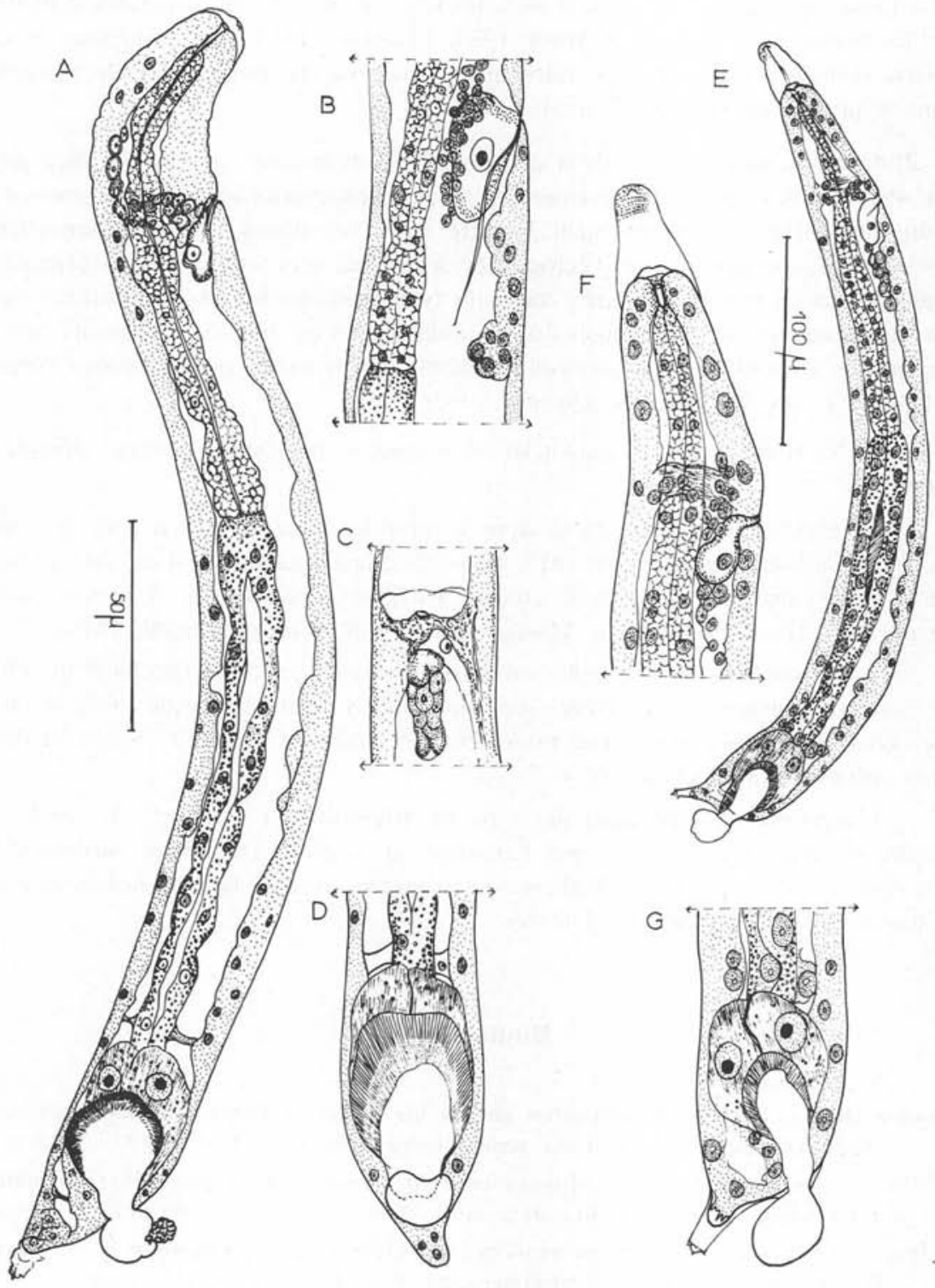

Fig. 3. - A) Larve au stade II ; B) très jeune larve $q$ du $2^{\text {e }}$ stade montrant la position de l'ébauche génitale ; C) sinus excréteur vu de face, au $2^{\circ}$ stade; D) région caudale du $2^{\circ}$ stade, vue de face ; F) larve en mue II, région antérieure ; E) idem, vue générale ; G) idem, région postérieure (A, B, C, D, F, G, éch. $50 \mu ; \mathrm{E}$ : éch. $100 \mu$ ) 
stade soit à l'origine des ornementations de l'adulte; il suffit, en effet, pour passer de l'un à l'autre d'imaginer, en accord avec les lois d'évolution des structures céphaliques chez les Spirurida (Chitwood et Wehr 1934, Chabaud, 1955), une réduction du cadre sur l'axe médian entraînant entre autres une disparition des pointes dorsale et ventrale, et une hypertrophie sur l'axe latéral.

2) Comparaison avec les deux cycles de Diplotriaeninae connus. La biologie des deux sous-familles morphologiquement proches, Diplotriaeninae et Dicheilonematinae, est du même type: développement larvaire lent chez divers insectes essentie!lement broyeurs, larves organisées dès l'éclosion et armées de crochets et pointes céphaliques, œsophage passant par un type très voisin du type rhabditoïde, forme infestante trapue. Tous ces caractères sont les mêmes que ceux des cycles de Spirurides primitifs, tels, par exemple que chez Oxyspirura mansoni (Cobbold 1879) parmi les Thelazidae (Schwabe 1951), et chez les Rictulariidae (Quentin 1969). férences :

Entre les trois cycles de Diplotriaenidae connus, il existe, en outre, quelque dif-

- Le crochet céphalique de la larve du premier stade est dorsal chez $D$. translucidus et D. bargusinica Skjrabin, 1917, (Diplotriaeninae) tandis qu'il est latéral gauche chez $S$. tendo ; une telle position du crochet a déjà été observée par Anderson en 1959 chez un autre Dicheilonematinae, Monopetalonema alcedinis (Rudolphi, 1819).

- L'encapsulement des larves est plus ou moins précoce, facultatif ou obligatoire : chez $D$. bargusinica les larves sont encapsulées pendant presque toute la vie larvaire ; chez D. translucidus, l'encapsulement est facultatif ; chez $S$. tendo, seules les formes infestantes sont encapsulées.

- L'organisation céphalique de la forme infestante est variable ; $S$. tendo, par exemple, se caractérise aisément par l'existence d'un cadre cuticulaire péribuccal ; en outre, cette espèce apparaît particulièrement primitive avec sa bouche hexagonale et la persistance des papilles labiales internes.

\section{Bibliographie}

Anderson (R. C.), 1957. - Observations on the life cycles of Diplotriaenoides translucidus Anderson and members of the genus Diplotriaena. Can. J. Zool., 35, 15-24.

-, 1959. - The morphology of Monopetalonema alcedinis (Rudolphi, 1819) (Nematoda: Filarioidea) including its first-stage larva. Can. J. Zool., 37, 609-614.

-, 1962. - On the development, morphology, and experimental transmission of Diplotriaena bargusinica (Filarioidea: Diplotriaenidae). Can. J. Zool., 40, 1175-1186.

Blanc (G.-R.), 1919. - Sur quelques espèces du genre Diplotriaena Raillet et Henry. Archiv. Parasit., 22, 546-556.

Снaвaud (A.-G.), 1955. - Remarques sur le cycle évolutif des Filaires du genre Diplotriaena et redescription de D. monticelliana (Stossich 1890). Vie et milieu, 6, 342-347. 


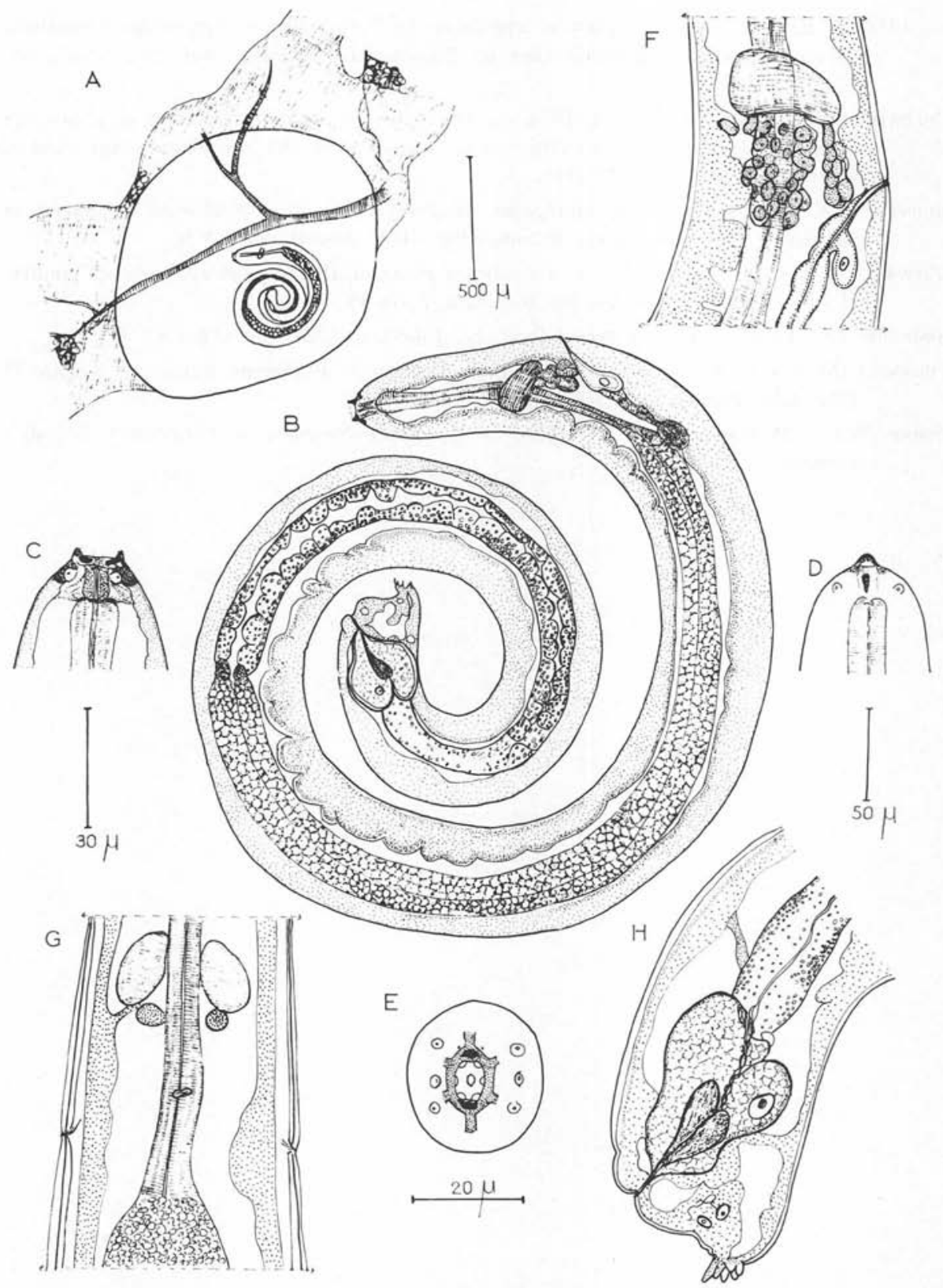

Fig. 4. - Forme infestante: A) travée de tissu adipeux renfermant une capsule avec une forme infestante ; B) allure générale ; C) région céphalique, vue latérale ; D) idem, vue médiane ; E) idem, vue apicale; F) région cervicale, vue latérale; G) idem, vue ventrale; H) région caudale, vue latérale (A, éch. $500 \mu$; B, éch. $50 \mu ; \mathrm{C}, \mathrm{D}, \mathrm{F}, \mathrm{G}, \mathrm{H}$, éch. $30 \mu$; E, éch. $20 \mu$ ) 
—, 1955. - Remarques sur la symétrie céphalique des Nématodes et hypothèses concernant l'évolution de cette symétrie chez les Phasmidiens parasites. Bull. Soc. Zool., 60, 314-323.

ChITwOod (B. G.) et WeHR (G. E.), 1934. - The value of cephalic structures as characters in nematode classification, with special reference to the superfamily Spiruroidea. Zeitsch. F. Parasit., 7, 273-335.

QUENTIN (J.-C.), 1969. - Cycle biologique de Pterygodermatites desportesi (Chabaud et Rousselot, 1956) Nematoda Rictulariidae. Ann. Parasit. 44, 47-58.

Schwabe (C. W.), 1951. - Studies on Oxyspirura mansoni, the tropical eyeworm of poultry. II Life history. Pacific Science Honolulu, 5, 18-35.

SonIN (M. D.), 1968. - Osnovi nematologii, 21, Filariata, Acad. Sc. U.R.S.S., 390 pp.

Tubangui (M. A.), 1934. - Nematodes in the collection of Philippine Bureau of Science II Filaroidea. Philippine J. Sci., 55, 115-122.

Yorke (W.) et MAPlestone (P. A.), 1926. - Nematode Parasites of Vertebrates, 536 pp., London. 